\title{
DEVELOPMENT OF A COMPUTATIONAL EXPERIMENT FOR THE PARALLELING OF THE MODIFIED BRANCH AND BOUND METHOD FOR THE PROBLEM FOR THE APPOINTMENT OF PROCEDURES TO PATIENTS IN SANATORIUM
}

\author{
Anna Danylchenko \\ Department of computer engineering \\ Zhytomyr State Technological University \\ 103 Chernyahivskiy str., Zhytomyr, Ukraine, 10005 \\ kissann@ukr.net \\ Svetlana Kravchenko \\ Department of Software Systems \\ Zhytomyr state technological university \\ 103 Chernyahivskiy str., Zhytomyr, Ukraine, 10005 \\ kravchenko-sveta2012@yandex.ua
}

\begin{abstract}
For the matching problem with vanishing arcs, an optimal algorithm based on the branch and bound method was developed to find the maximum matching in a bipartite graph [1].

The algorithm takes into account the limits of compatibility procedures. The calculated experiment is aimed at proving the feasibility of paralleling of the optimal algorithm for solving the problem of scheduling the reception of medical procedures by patients for use in the sanatoriums of Ukraine.

The experiment was carried out on computing platforms of different configurations with different computing power: a different number of processor cores, different amounts of memory, etc.

Estimated minimum time scheduling, received at the computer platform with the maximum number of PCs is involved. Estimated time scheduling algorithm paralleling by using modifications of the branch and bound method is directly proportional to the number of vertices of a bipartite graph (which is equal to the sum of the number of procedures and the number of patients), the number of assigned procedures and restrictions.
\end{abstract}

Keywords: bipartite graphs, branch and bound method, method of exhaustive search.

\section{Introduction}

The appointment of procedures in modern sanatorium and medical institutions is a complex process. This process must take into account a sufficiently large number of factors, the main of which are:

- list of procedures prescribed by the doctor; the operating time of the medical treatment room;

- capacity of the medical treatment room (one procedure can be taken by several patients at a time);

- duration of the procedure (for different procedures the duration of the procedure is different);

- duration of the technical break between the procedures; compatibility of procedures (the patient can't simultaneously take several procedures). Also, the schedule is imposed an additional restriction - the patient can't has the following procedure less than a short time after the previous one, for each pair of procedures the value of the compatibility time may vary.

The task of scheduling procedures for patients of a sanatorium is considered in the article [1]. Such problems are successfully solved by the mathematical apparatus of the theory of schedules - a section of applied mathematics that studies models of ordering of works and 
methods of scheduling [2]. The task of appointment of the procedures to patients in the sanatorium is reduced to an extended task of finding the maximum matching in a bipartite graph. An optimal algorithm for its solution is developed on the basis of the known of branch and bound method [1].

The tasks of scheduling can be interpreted and how the tasks of allocating resources (under the resources in the broad sense, mean material, time resources, etc.) with given constraints (by the number of resources, the time for their creation, processing, etc.) [3]. The source [4] describe the task of scheduling training sessions for schools, universities, etc.

From the NP-completeness of the problem "On matching with vanishing arcs", which is proved in [5], it follows that it does not lend itself to efficient, accurate methods.

It is known that any NP-complete problem can be solved by the method of complete search [6]. But at the same time, depending on the size of the problem, computing resources are needed and the time of its solution can be unacceptably large from a practical point of view.

The tasks of compiling the optimal schedule are often amenable to solving, using the modifications of the classical problem of matchings [7], various statements of which are given [8]. This problem consists in finding the minimum cost in an arbitrary weighted graph and in [9] it is solved in time $\mathrm{O}(\mathrm{n} \log (\mathrm{n}))$, where $\mathrm{n}$ - the order of the input matrix.

To optimize the process of full enumeration, the branch and bound method [6] is applied, which allows to reduce the set of admissible solutions by means of an effective search algorithm, as well as paralleling of computations.

It should be noted that for the branch and bound method the branching procedure and the procedure for finding the upper and lower bounds have the greatest difficulty.

Paralleling of the computation does not reduce the number of options that are analyzed, but only reduces the time required for this.

In $[10,11]$, algorithms for solving the classical matching problem are proposed, which make it possible to reduce the computational complexity of software implementations.

In [12], the authors develop an algorithm for solving the task of scheduling tasks by modifying the Hungarian algorithm.

In [13], a new method for solving the problem of weighted matching is described, the maximum matching in which is in time $\mathrm{O}\left(\mathrm{n}^{3}\right)$. In the future, it is planned to modify the problem of matching with "vanishing" arcs, adding weight to the arcs.

The aim of the computational experiment is to prove the feasibility of paralleling of the proposed modification of the branch and bound method for solving the problem of matching with vanishing arcs for its further application in the sanatoriums of Ukraine.

\section{Materials and Methods}

Since the software product is aimed at the final customer (sanatorium "Denyshi" in the Zhytomyr region), the general formulation of the problem is as follows:

1. To develop a program that ensures the preservation of the following data:

- information about patients and saving the data archive;

- full characteristics of procedures and time of compatibility of the procedures;

- information about doctors, their specializations and weighting coefficients;

- weekends and holidays.

2. The program must ensure the following functions and calculations:

- add, change, delete, sort, print and search data for all listed items;

- fixing the patient to a certain doctor and calculating the burden of doctors;

- the appointment of a certain number of procedures by the doctor to the patient;

- formation of the patient's schedule taking into account the capacity of the medical treatment rooms.

3. The system should ensure the formation of a schedule in two modes:

- formation of the patient's schedule immediately after the appointment (manual mode);

- automatic scheduling for all patients. 
4. Ability to edit the schedule.

5. Calculation, viewing and printing of general and free limits of doctors on the appointment of procedures.

6. Calculation, viewing and printing of the reserve of procedures and the total number of appointed procedures.

7. Calculation and printing of capacity of medical treatment rooms on a certain date.

To achieve the aim of the experiment it is necessary:

1. To estimate the time costs for performing calculations on various computing platforms having different computational powers: different number of processor cores, different amount of memory, and so on.

2. To compare the results and determine the efficiency and appropriateness of the paralleling process. The efficiency criterion is the minimization of the execution time for the calculation of the maximum matching in a bipartite graph with vanishing arcs.

The experiment was conducted using the author's problem-oriented tools - NetRemoting program.

NetRemoting program is designed for automated scheduling of procedures for patients of a sanatorium. In addition, it provides: calculation of doctors' work, calculation, viewing and printing of general and free limits of doctors, calculation, viewing and printing of the reserve procedures and the total number of appointed procedures, calculation of capacity of the medical treatment rooms.

The program for the automated formation of the schedule for procedures for the patients of the sanatorium (NetRemoting) is designed for performance on an IBM PC and runs under the operating system Windows 7 or higher.

PC must have at least $512 \mathrm{MB}$ of RAM, a graphics card with $64 \mathrm{MB}$ or more RAM, SVGA monitor with a resolution of at least $800 \times 600$ pixels, at least $50 \mathrm{MB}$ of free disk space.

The program consists of two modules: a server module that controls the execution of calculations and a client module that runs on separate PCs to calculate parallel operations. The program solves the problem of scheduling procedures for patients of the sanatorium with a modified branch and bound method. The architecture of the program complex is shown in Fig. 1.

The computational experiment was carried out on a series of random conditions of the problem generated by the program. Input parameters (Table 2) were random (registered, but uncontrolled). The initial parameter for carrying out the computational experiment is $\mathrm{t}_{\mathrm{c}}^{\mathrm{pr}}-\mathrm{the}$ predicted time for the formation of the schedule under given conditions. The characteristics of computers were chosen taking into account the increase in the number of processor cores for clarity of the algorithm paralleling. The number of tests and the input parameters correspond to the data in Table 1, 2.

Table 1

The characteristics of computers that were used to conduct a comparative computing experiment

\begin{tabular}{|c|c|c|c|c|}
\hline $\begin{array}{l}\text { Number of } \\
\text { the computing platform }\end{array}$ & Microprocessor name & Number of cores & Clock frequency & RAM \\
\hline 1 & Pentium 4 & 1 & $2,42 \mathrm{GHz}$ & $512 \mathrm{MB}$ \\
\hline 2 & Intel Celeron E3300 & 2 & $2,50 \mathrm{GHz}$ & $2 \mathrm{~GB}$ \\
\hline 3 & Intel Core i5-3570K & 4 & $3,4 \mathrm{GHz}$ & $4 \mathrm{~GB}$ \\
\hline
\end{tabular}




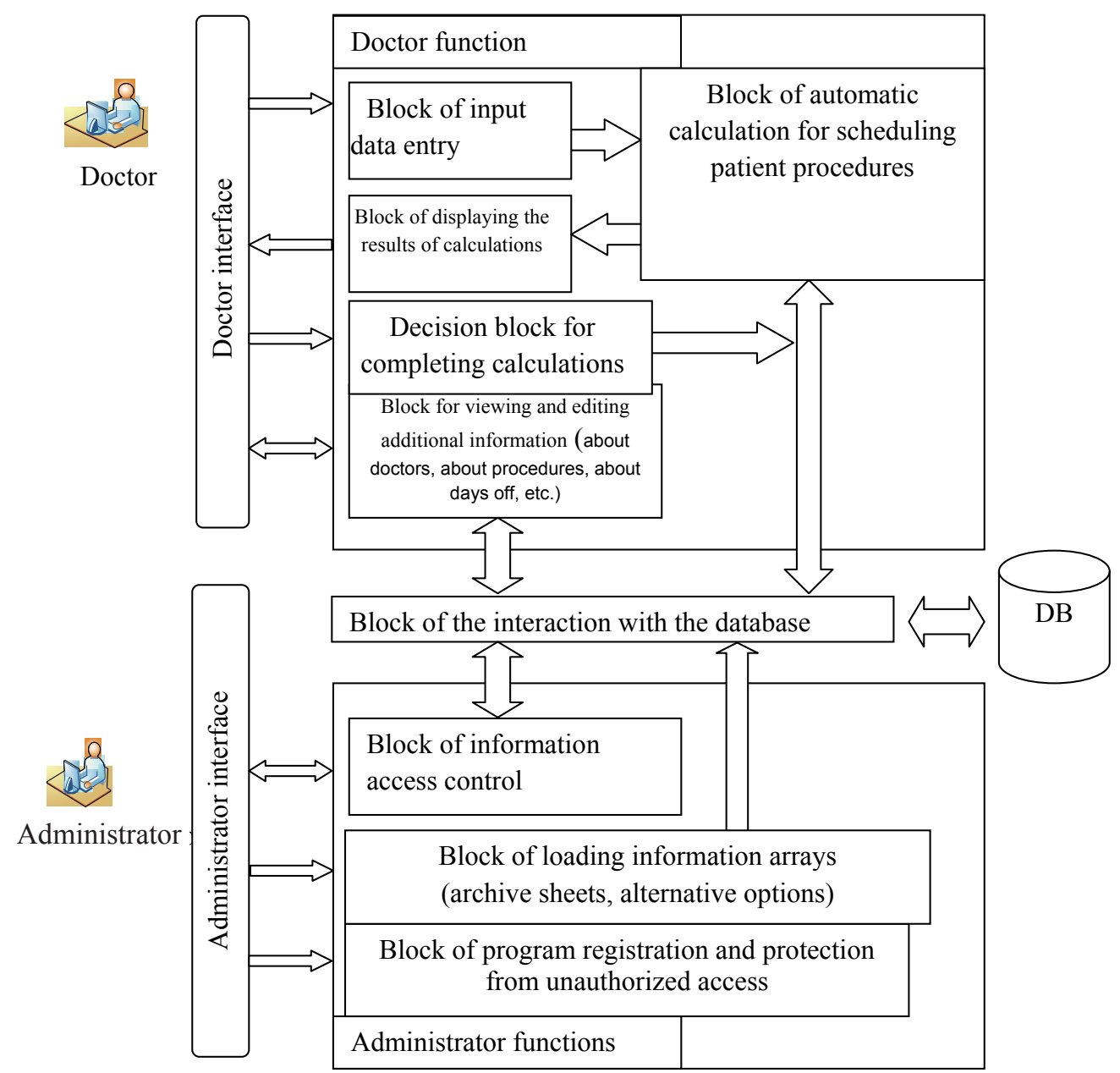

Fig. 1. The architecture of the software product of automatic scheduling of medical procedures by patients of the sanatorium

Table 2

List of input parameters of the computational experiment

\begin{tabular}{|c|c|c|c|}
\hline $\begin{array}{c}\text { Parameter } \\
\text { number }\end{array}$ & Designation & Parameter name & Parameter type \\
\hline 1 & $\mathrm{k}_{\mathrm{tp}}$ & number of treatment procedures & deterministic \\
\hline 2 & $\mathrm{k}_{\mathrm{p}}$ & number of patients & random \\
\hline 3 & $\mathrm{k}_{\mathrm{ap}}$ & number of appointed procedures & random \\
\hline 4 & $\mathrm{k}_{\mathrm{i}, \mathrm{j}}$ & restrictions on procedures & deterministic \\
\hline
\end{tabular}

The initial parameter for conducting a comparative computing experiment is the time $t_{\tilde{n}}$ spent on making calculations for scheduling the reception of treatment procedures by the patients of the sanatorium using NetRemoting program 


\section{Experimental procedures}

Generalized instructions for the use of software in a computational experiment:

1. Run the software on the server, select the required number of clients and wait for their connection (Fig. 2).

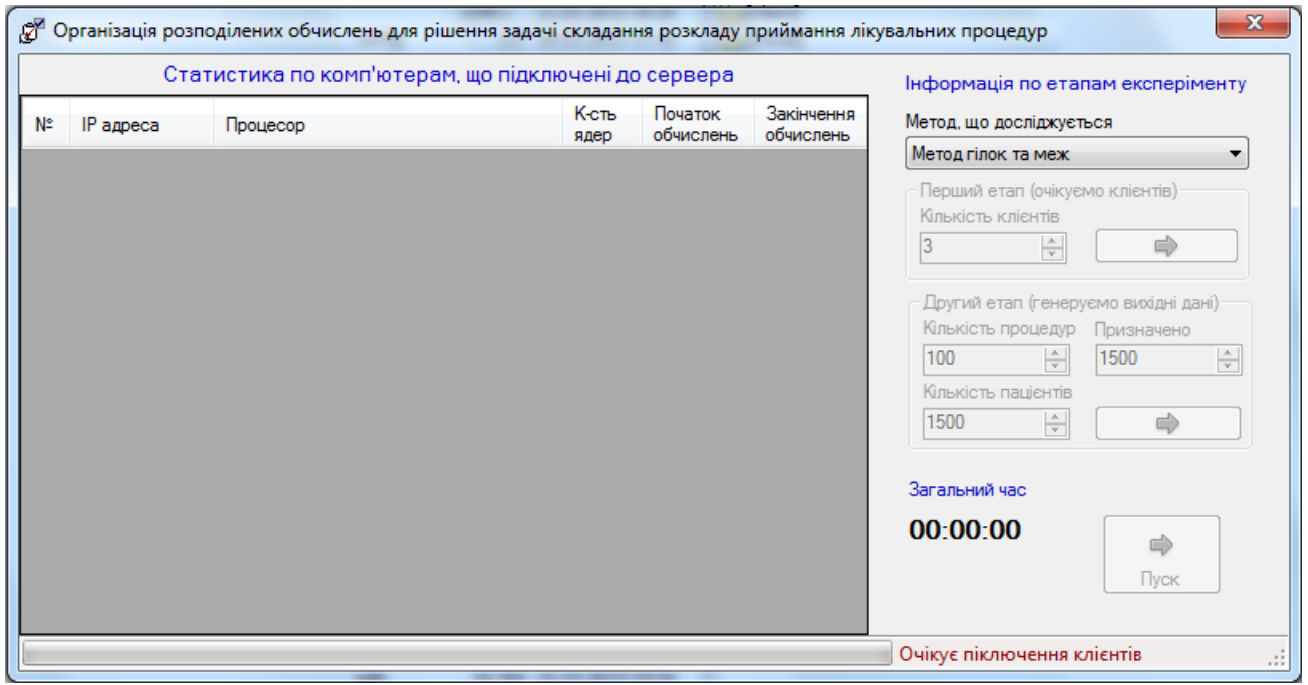

Fig. 2. The main window of the server module

2. After connecting all the clients, the server goes into the output data generation mode, selects the required number of procedures, people, appointed procedures and presses the button, the server generates the data. The window for generating input data is shown in Fig. 3.

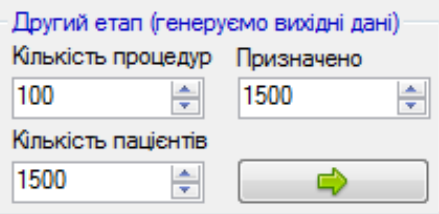

Fig. 3. Output data generation window

The server goes into the ready for calculations mode (Fig. 4).

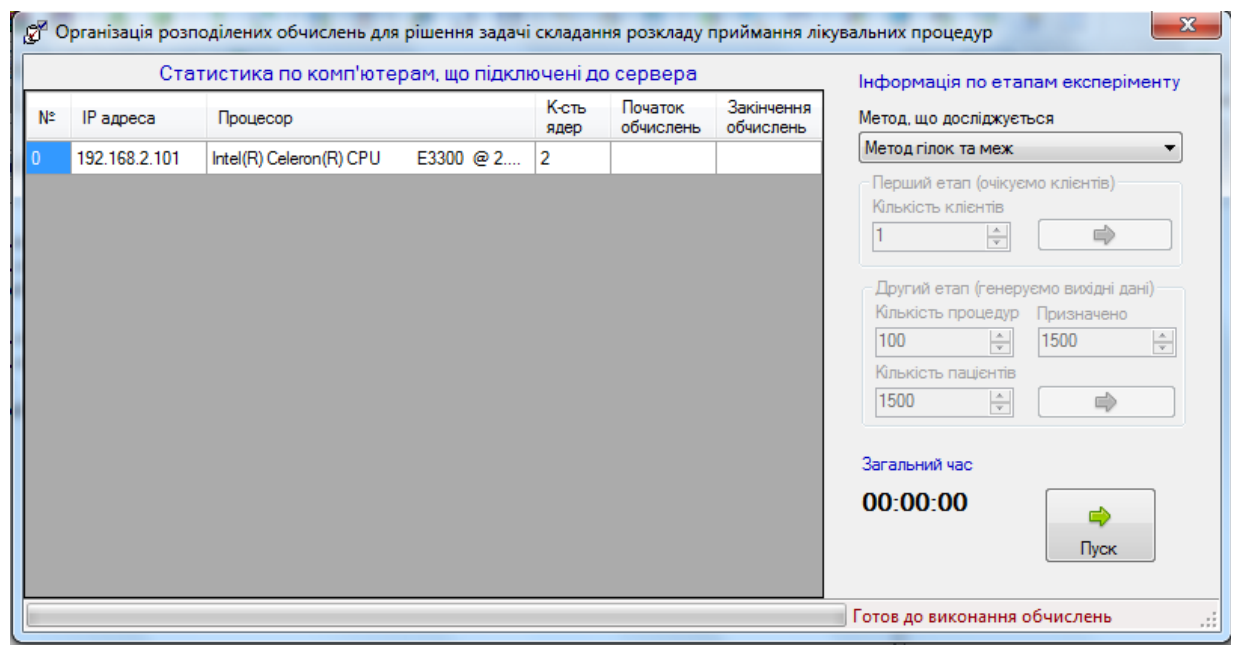

Fig. 4. Readiness window of the server module for calculations 
3. Get the result (Fig. 5).

$$
\begin{aligned}
& \text { Загальний час } \\
& 0: 0 \text { : } 49
\end{aligned}
$$

Fig. 5. The window with the result of calculation $t_{c}{ }^{p r}$

For carrying out of computing experiment computers with characteristics according to Table 1 were used. Three computing platforms were formed (Fig. 6-8).

\begin{tabular}{|cc|}
\hline \multicolumn{1}{|c|}{} & Intel Celeron E3300 \\
\cline { 2 - 2 } core 1 & control process \\
\cline { 2 - 2 } & \\
core 2 & working process \\
\cline { 2 - 2 } &
\end{tabular}

Fig. 6. The structure of the processor, which was used on the first computing platform

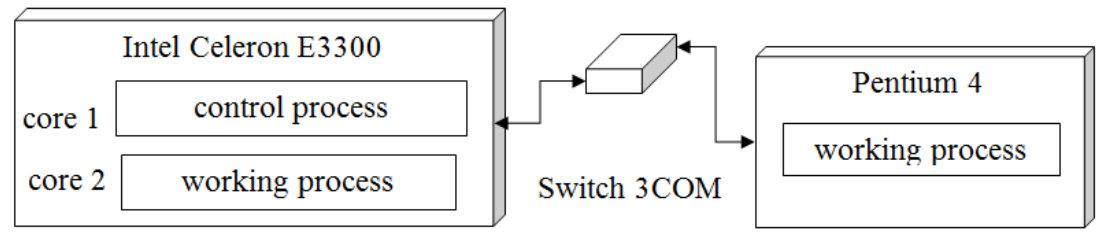

Fig. 7. The structure of the processor, which was used on the second computing platform

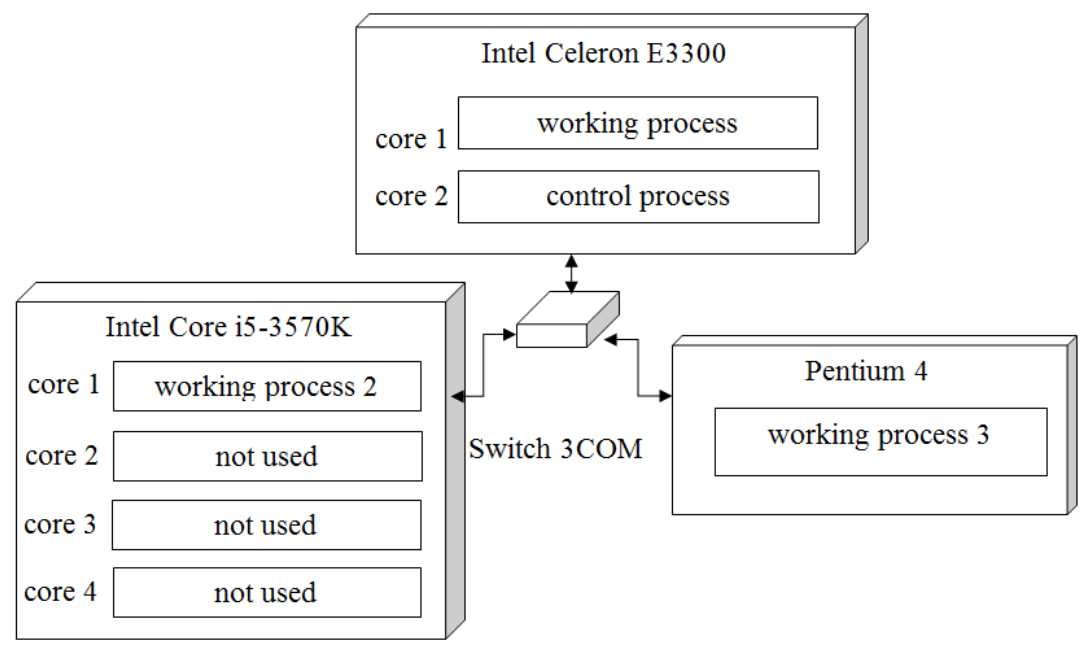

Fig. 8. The structure of the processor, which was used on the third computing platform

In each test (Table 3), a time was measured to perform calculations by modifying the branch and bound method for various computational platforms shown in Fig. 6-8.

As can be seen from the data in Table 3, the value of the input parameters increases with the number of the test. Also, in accordance with the increase in the values of the parameters, the time for the formation of the schedule increases. The computational experiment was carried out on a series of random conditions of the problem generated by the program. Input parameters for the experiment were registered, but uncontrolled. 
Table 3

Results of tests of computing experiment

\begin{tabular}{ccccccc}
$\begin{array}{c}\text { Test } \\
\text { number }\end{array}$ & \multicolumn{3}{c}{ Input parameters } & \multicolumn{3}{c}{ The predicted time of the schedule formation } \\
& $\mathbf{k}_{\mathrm{tp}}$ & $\mathbf{k}_{\mathrm{p}}$ & $\mathbf{k}_{\mathrm{ap}}$ & The first platform & The second platform & The third platform \\
\hline 1 & 74 & 1584 & 2974 & 71,2 & 37,4 & 23,8 \\
2 & 74 & 1312 & 3217 & 91,5 & 48,8 & 31,2 \\
3 & 80 & 1723 & 3343 & 112,4 & 59,2 & 38,7 \\
4 & 80 & 1784 & 3578 & 115,8 & 60,1 & 40,4 \\
5 & 85 & 1837 & 3724 & 137 & 71,7 & 47 \\
- & - & - & - & - & - & - \\
86 & 129 & 8719 & 51622 & 412,2 & 209,3 & 138,1
\end{tabular}

\section{Discussion}

During the experiment, the following results were obtained:

1. The predicted time of scheduling using the algorithm of paralleling of the modification of the branch and bound method is directly proportional to the number of vertices of the bipartite graph (which is equal to the sum of the number of procedures and the number of patients), the number of assigned procedures and restrictions.

2. The predicted minimum time for scheduling is obtained on a computer platform with the maximum number of involved PCs.

3. The maximum reduction in time is 19.1 times compared to the 86 th test for the third platform with the 86th test for the first platform given in Table 3.

Thus, the aim of the experiment is achieved and the expediency of paralleling of the proposed modification of the branch and bound method for solving the problem of matching with vanishing arcs for application in sanatoriums of Ukraine is achieved.

\section{Conclusions}

Using the developed NetRemoting software, a comparative computational experiment was performed, which allowed estimating the temporal characteristics of the optimal algorithm for solving the matching problem with vanishing arcs on different computational platforms and comparing them. Based on the results of the experiment, it is possible to draw conclusions about the advisability of paralleling of the solution of the matching problem with vanishing arcs by an optimal algorithm. The modified branch and bound method shows the stability of reducing the time for scheduling procedures by patients with increasing computing power of the $\mathrm{PC}$, for example, at $\mathrm{k}_{\mathrm{tp}}=85, \mathrm{k}_{\mathrm{p}}=1837, \mathrm{k}_{\mathrm{ap}}=3724$, the time of solving the problem on the third platform is reduced by 3 times in comparison with the first computing platform.

The practical value of researches lies in the possibility of their use in the development and application of scheduling systems and operational management in the treatment process. Researches are also applicable in the development of control systems for flexible automated systems for enterprises with a discrete nature of production.

The authors would like to further add weights to each arc and solve the problem of matching with "vanishing" arcs in a modified form and to conduct an experiment of paralleling of its solution.

A promising direction for further research is the modification of known methods (genetic, ant colony, etc.) to solve the problem of scheduling procedures for patients of the sanatorium, as well as paralleling of these methods and comparison of the results with obtained in this research. 


\section{References}

[1] Danilchenko, A. (2012). Optimal algorithm for solving the problem of matching with vanishing arcs. Science news, 6, 46-54.

[2] Lupin, S. A., Milehina, T. V. (2007). The method for solving scheduling problems, focused on cluster computing systems. Proceedings of the universities. Ser. Electronics, 6, 63-69.

[3] Gafarov, E. (2007). Hybrid algorithm for solving the problem of minimization of summarized delay for one device. Information technology, 1, 30-37.

[4] Tymofijeva, N. K., Grycenko, V. I. (2011). Solving the problem of scheduling theory planning by structural alphabet search algorithm and hybrid. Upravliaiushchie sistemy i mashiny, 3, 21-36.

[5] Panishev, A., Danilchenko, A., Danilchenko, A. (2012). The problem of matching with the disappearing "arcs". Simulation and informational technologies, 63, 75-81.

[6] Papadimitriou, C. H., Steiglitz, K. (1998). Combinatorial Optimization: Algorithms and Complexity. Dover Publications, 528.

[7] Biro, P., Manlove, D. F., Mittal, S. (2010). Size versus stability in the marriage problem. Theoretical Computer Science, 411 (16-18), 1828-1841. doi: 10.1016/j.tcs.2010.02.003

[8] Mugurel, I. A. (2014). Practical Algorithmic Optimizations for Finding Maximal Matchings in Induced Subgraphs of Grids and Minimum Cost Perfect Matchings in Bipartite Graphs. Theory and Applications of Mathematics \& Computer Science, 4 (1), 1-13.

[9] Gelain, M., Pini, M., Rossi, F., Venable, K., Walsh, T. (2013). Local Search Approaches in Stable Matching Problems. Algorithms, 6 (4), 591-617. doi: 10.3390/a6040591

[10] Sonkin, D. M. (2009). Adaptive algorithm of distributing orders for taxi service. Bulletin of the Tomsk Polytechnic University, 315 (5), 65-69.

[11] Li, W., Patrikeev, E. M., Xiao, D. (2015). A DNA Algorithm for the maximal matching problem. Automation and Remote Control, 76 (10), 1797-1802. doi: 10.1134/s0005117915100070

[12] Sergiyenko, A. M., Simonenko, V. P., Simonenko, A. V. (2016). An enhanced scheduling algorithm for task planners in heterogeneous distributed computing systems. System Research and Information Technologies, 2, 20-35. doi: 10.20535/srit.2308-8893.2016.2.03

[13] Matsiy, O. B., Morozov, A. V., Panishev, A. V. (2016). A Recurrent Algorithm to Solve the Weighted Matching Problem. Cybernetics and Systems Analysis, 52 (5), 748-757. doi: 10.1007/s10559016-9876-4 